ISSN 0103-5150

Fisioter. Mov., Curitiba, v. 24, n. 4, p. 683-688, out./dez. 2011

Licenciado sob uma Licença Creative Commons

\title{
Ansiedade e parâmetros funcionais respiratórios de idosos praticantes de dança
}

\author{
Anxiety and functional respiratory parameters of elders dancers
}

\author{
Adriana Coutinho de Azevedo Guimarães ${ }^{[a]}$, Aline Pedrini ${ }^{[b]}$, Darlan Laurício Matte ${ }^{[c]}$, \\ Fernanda Guidarini Monte ${ }^{[\mathrm{d}]}$, Silvia Rosane Parcias $^{[\mathrm{e}]}$
}

[a] Doutoranda da Faculdade de Motricidade Humana da FMH-UTL do Departamento de Educação Física, Núcleo de Atividade Física, Saúde e Gerontomotricidade (NAFISGE/LADEHU) da Universidade do Estado de Santa Catarina (UDESC), Florianópolis, SC - Brasil, e-mail: nanaguim@terra.com.br

[b] Acadêmica do Curso de Fisioterapia pela UDESC, Florianópolis, SC - Brasil, e-mail: alinepedrinii@gmail.com

[c] Doutorando em Ciências Médicas, Mestre em Ciências do Movimento Humano e docente do Departamento de Fisioterapia da UDESC, Florianópolis, SC - Brasil, e-mail: darlan@udesc.br

[d] Doutoranda em Atividade Física e Saúde da Universidade Federal Santa Catarina (UFSC), Florianópolis, SC - Brasil, e-mail: fernandamonte@hotmail.com

[e] Doutora em Neurociências, coordenadora do Núcleo de Atividade Física, Saúde e Gerontomotricidade (NAFISGE/ LADEHU) e professora orientadora da Universidade do Estado de Santa Catarina (UDESC), Florianópolis, SC - Brasil, e-mail: sil_pa13@hotmail.com

\section{Resumo}

Introdução: A ansiedade é um diagnóstico comum encontrado em idosos e se associa comumente a anormalidades respiratórias. Ligado ao envelhecimento, encontra-se também o sedentarismo, o qual é um importante fator de risco para diversas patologias. A prática de atividade física regular melhora a ansiedade e a condição cardiorrespiratória. Entre os tipos de atividades físicas, a dança destaca-se como uma boa opção para os idosos. Objetivo: Descrever os parâmetros respiratórios de idosas praticantes de dança quando comparadas com idosas sedentárias e a influência da prática regular de dança sobre a ansiedade. Materiais e métodos: Foram avaliadas 18 idosas, divididas em dois grupos: praticantes de dança (grupo G1) e sedentárias (grupo G2). Nos grupos G1e G2, foram aplicados o inventário de ansiedade traço-estado IDATE, a cirtometria, a espirometria e a manovacuometria, além de ser utilizado o questionário de Baecke modificado para idosos, apenas para o grupo G2, a fim de garantir o sedentarismo dos participantes. Foi realizada a 
estatística descritiva para sintetizar os dados. Resultados: As participantes do grupo G1 apresentaram valores superiores em relação à ansiedade-estado e à pressão expiratória máxima. A cirtometria em nível basal foi maior nas participantes do grupo G1, enquanto em nível xifoide foi maior nas idosas do grupo G2. Todas as participantes obtiveram valores de espirometria dentro da normalidade, sem diferenças entre os grupos. Conclusão: Observaram-se nas idosas que praticam dança de forma regular menor nível de ansiedade-traço, valores adequados de pressão expiratória máxima e maior valor na cirtometria em nível basal.

Palavras-chave: Ansiedade. Respiração. Idosos. Dança.

\section{Abstract}

Introduction: Anxiety is a common diagnosis found in elderly and is commonly associated with breathing abnormalities. Associated with aging, it is also found a sedentary lifestyle, which is an important risk factor for diseases. The practice of regular physical activities improves anxiety and cardiorespiratory condition. Between the types of physical activities, dance stands out as a good option for elderly. Objective: To describe the influence of regular dance on anxiety and respiratory parameters of elderly dancers when compared with sedentary elders. Methodology: Were evaluated 18 elders, divided into two groups: dancers (G1 group) and sedentary (G2 group). In G1 and G2 groups it was applied the State-Trait Anxiety Inventory, cirtometry, spirometry and manovacuometry, in addition of the questionnaire of Baecke modified to elders, only for the group G2, in order to ensure the sedentary lifestyle of the participants. Descriptive statistics were used to synthesize the data. Results: The participants in group G1 had higher values in relation of state-anxiety and maximal expiratory pressure. Cirtometry at basal level was higher in participants of group G1, while the xiphoid level was greater in group G2. All participants had spirometry values within the normal ranges, with no differences between groups. Conclusion: We observed on elders who practice dance regularly lower level of trait-anxiety, appropriate values of maximal expiratory pressure and higher values on cirtometry at basal level.

Keywords: Anxiety. Breath. Elders. Dance.

\section{Introdução}

Nos países em desenvolvimento, a transição epidemiológica com o envelhecimento populacional tem sido motivo de preocupação. 0 aumento da prevalência de idosos vem acontecendo de forma muito rápida. 0 número de idosos no Brasil passou de 3 milhões em 1960 para 20 milhões em 2008. Consequentemente, houve também um aumento no número de doenças, o que é comum nessa idade (1).

Estudos mostram que um dos diagnósticos mais encontrados nessa população é o transtorno de humor acompanhado de ansiedade (15,4\% dos casos), que se caracteriza por um sentimento vago e desagradável de medo e apreensão $(2,3)$.

Associam-se comumente com a ansiedade as anormalidades respiratórias, nas quais $50 \%$ a $60 \%$ das pessoas descrevem sintomas de hiperventilação, apresentado durante as crises de dificuldades em respirar, a taquicardia, a sensação de sufocação e a tontura (4).
As alterações na função pulmonar relacionadas com a idade são clinicamente relevantes, visto que uma função pulmonar precária é associada a taxas elevadas de mortalidade. Com o avanço da idade, ocorre redução da mobilidade da caixa torácica, da elasticidade pulmonar e dos valores das pressões inspiratória e expiratória máximas, o que pode gerar sintomas de dificuldades respiratórias $(5,6)$.

Associado ao envelhecimento geralmente encontra-se o sedentarismo, considerado um importante fator de risco para doenças. A prática de atividade física regular melhora a ansiedade, a depressão e gera adaptações fisiológicas, como a melhora da função pulmonar, tornando os idosos mais independentes, autônomos e sadios (7-9).

Entre os tipos de atividade física, a dança destaca-se como uma boa opção para os idosos, pois parece reduzir angústias, medos e inseguranças, levando a um bem-estar físico, social e psicológico e melhorando a qualidade de vida dessa população. Estudos 
realizados sobre a prática da dança de salão em mulheres idosas demonstraram que houve melhoras tanto na aptidão funcional quanto na qualidade de vida das participantes $(10,11)$.

Baseado no exposto, este estudo teve por objetivo descrever influência da prática regular de dança sobre a ansiedade e sobre os parâmetros respiratórios de idosos praticantes de dança quando comparados com idosos sedentários.

\section{Metodologia}

O estudo de caráter descritivo interpretativo teve uma população composta por 30 idosas que praticam a dança de salão adaptada à terceira idade, no Centro de Atenção à Terceira Idade /CATI - São José/ SC, e por idosas sedentárias moradoras da mesma comunidade.

O estudo foi aprovado pelo Comitê de Ética em Pesquisa Envolvendo Seres Humanos da UDESC, sob protocolo número 96/2010. Todos os participantes foram esclarecidos acerca dos procedimentos e dos objetivos do estudo e assinaram o Termo de Consentimento Livre e Esclarecido.

Das 30 idosas selecionadas, 12 foram excluídas por não conseguirem completar as avaliações propostas no presente estudo, ficando a amostra composta por 18 idosas, com idade de $66 \pm 5,1$ anos, escolhidas de forma intencional não probabilística, divididas em dois grupos distintos de acordo com o resultado obtido pelo questionário de Baecke modificado para idosos (12), com pessoas recrutadas a partir da comunidade de São José: grupo G1 - 9 praticantes de dança de salão adaptada à terceira idade e grupo G2 - 9 idosas sedentárias.

Foram adotados como critérios de inclusão para os grupos: ter idade igual ou superior a 60 anos; não possuir histórico de tabagismo, de cardiopatias ou de pneumopatias; ter capacidade para realizar as avaliações do estudo; não estar fazendo uso de medicamentos (imunossupressores) que interfiram na avaliação e assinar o termo de consentimento livre e esclarecido. Para o grupo G1: realizar o programa de dança há pelo menos dois anos, com frequência mínima de $75 \%$ nas aulas. Para o grupo G2: escore no questionário de Baecke modificado para idosos menor que 9,4, o que irá confirmar o sedentarismo das participantes.

Os instrumentos de pesquisa empregados foram: espirômetro EasyOne ${ }^{\circledR}$ da marca NDD; balança digital da marca Filizola com estadiômetro da marca Sanny (precisão de 100 g e 1 mm); manovacuômetro digital modelo MVD 300 da marca Globalmed, fita métrica da marca Prim, inventário de ansiedade traçoestado (13) e questionário de Baecke modificado para idosos (12).

Para investigar a presença ou não de ansiedade traço-estado das participantes, utilizou-se o Inventário de Ansiedade Traço-Estado (IDATE), que consiste em 20 afirmações sobre como o indivíduo se sente no exato momento do teste (ansiedade-estado) e outras 20 sobre como se sente geralmente (ansiedadetraço). A pontuação máxima obtida em cada uma das escalas é de 80, e a mínima, de 20 pontos; uma pontuação acima de 33 já indica níveis de ansiedade (13).

A avaliação antropométrica para obtenção da massa e estatura corporal dos indivíduos foi realizada em balança com estadiômetro, previamente calibrada, com o indivíduo descalço, em posição ereta e cabeça alinhada.

Os fluxos e os volumes aéreos foram mensurados por meio de um espirômetro previamente calibrado. Foi verificado o volume expiratório forçado no primeiro segundo em valor absoluto - em litros/segundo - e em percentual do previsto e a capacidade vital forçada. Os valores previstos foram os estabelecidos pela tabela de referência de Pereira, sendo que a espirometria seguiu as diretrizes da Sociedade Brasileira de Pneumologia e Tisiologia $(14,15)$.

As medidas de força da musculatura respiratória foram realizadas por meio de um manovacuômetro para conseguir os valores da pressão inspiratória máxima (PImáx) e da pressão expiratória máxima (PEmáx) em $\mathrm{cmH}_{2} \mathrm{O}$, as quais foram aplicadas com as idosas na posição sentada e com uso de clipe nasal. A PEmáx foi mensurada após uma expiração máxima esperando-se estar próximo ao volume residual, enquanto que a PEmáx foi após uma inspiração máxima esperando-se estar próximo à capacidade pulmonar total. Para cada variável, foram necessárias pelo menos três manobras reprodutíveis (com variação de valores menor que $20 \%$ ) e o maior valor alcançado foi o considerado para fins do estudo (16).

Para a cirtometria, utilizou-se uma fita métrica para obtenção de medidas das circunferências de três pontos anatômicos: prega axilar, processo xifoide e linha umbilical. Foi solicitado que as participantes executassem uma expiração máxima seguida de uma inspiração máxima duas vezes, sendo considerada a média dos dois valores obtidos (17). 
Para assegurar que o grupo G2 era formado por indivíduos sedentários, aplicou-se, sob a forma de entrevista, o questionário de Baecke modificado para idosos, o qual investiga as atividades físicas habituais praticadas nos últimos 12 meses. É composto por 16 questões, todas com respostas variando entre muito ativo e inativo e com pontuações específicas. Uma pontuação menor que 9,4 classifica o indivíduo como inativo (12).

Os instrumentos utilizados foram o Inventário de Ansiedade Traço e Estado, seguido da avaliação antropométrica, espirometria, cirtometria e por último a manovacuometria. Para o grupo G2, também foi realizado inicialmente o questionário de Baecke modicado para idosos. As avaliações acontecerem de forma individual, na clínica escola do Centro de Ciências da Saúde e do Esporte (CEFID), em sala previamente reservada, seguindo os critérios de biossegurança, com tempo médio de 60 minutos por participante.

Para a análise estatística, confeccionou-se uma planilha eletrônica no programa Excel 2007, utilizando a estatística descritiva para sintetizar os dados, permitindo, dessa forma, que se tivesse uma visão global da variação dos valores obtidos.

\section{Resultados}

Na Tabela 1 estão apresentadas as características dos grupos do estudo. Observa-se pouca diferença em relação às variáveis idade, peso e estatura, podendo-se considerar os grupos como semelhantes.

Na Tabela 2 estão apresentados os níveis de ansiedade traço-estado dos grupos estudados. As idosas do grupo G1 apresentaram menores níveis de ansiedade-traço e maiores níveis de ansiedade-estado em relação às do grupo G2.

As medidas de força muscular respiratória estão expostas na Tabela 3 .

Tabela 1 - Características da amostra

\begin{tabular}{lcc}
\hline & G1 & G2 \\
\hline Idade (anos) & $67 \pm 5,2$ & $65 \pm 5$ \\
Peso (kg) & $63 \pm 8,8$ & $65 \pm 11,9$ \\
Altura (cm) & $153 \pm 4.6$ & $159 \pm 5,4$ \\
\hline
\end{tabular}

Legenda: Média \pm DP.

Fonte: Dados da pesquisa.
As participantes do grupo G1 apresentaram valores de PEmáx. em normalidade com o valor previsto. Todas as participantes do grupo G2 mostraram valores de força muscular respiratória inferior ao grupo G1.

As medidas de cirtometria e espirometria estão representadas na Tabela 4. As participantes do grupo G2 apresentaram aumento na cirtometria a nível xifoide em relação às do grupo G1 e menor mobilidade abdominal. Não houve diferenças em termos percentuais em relação à mobilidade de nível axilar e à espirometria.

Tabela 2 - Níveis de ansiedade traço-estado

\begin{tabular}{lccc}
\hline & G1 & G2 & Diferença \\
\hline Ansiedade-traço & $31,7 \pm 8,7$ & $33,2 \pm 8,0$ & 1,5 \\
Ansiedade-estado & $33,1 \pm 5,9$ & $31,6 \pm 7,7$ & 1,5 \\
\hline
\end{tabular}

Legenda: Média \pm DP.

Fonte: Dados da pesquisa.

Tabela 3 - Medidas de força muscular respiratória

\begin{tabular}{lcc}
\hline & G1 & G2 \\
\hline PEmáx. & $-61 \pm 28,0$ & $-51 \pm 10,3$ \\
PEmáx. \% do previsto & 78 & 65 \\
PEmáx. & $67 \pm 22,9$ & $65 \pm 10,4$ \\
PEmáx. \% do previsto & 81 & 71 \\
\hline
\end{tabular}

Legenda: Média \pm DP.

Fonte: Dados da pesquisa.

Tabela 4 - Dados da cirtometria e da espirometria

\begin{tabular}{lcc}
\hline & G1 & G2 \\
\hline CVF (\%) & $84 \pm 14,1$ & $90,4 \pm 22,2$ \\
VEF $_{1}(\%)$ & $79,9 \pm 16,4$ & $90.1 \pm 19.7$ \\
CVF/VEF $_{1}(\%)$ & $0,80 \pm 0,01$ & $0,79 \pm 0,01$ \\
Linha Axilar (cm) & $4,4 \pm 1,3$ & $5 \pm 1,1$ \\
Xifoide (cm) & $2,4 \pm 1,3$ & $3,9 \pm 1,1$ \\
Basal (cm) & $3,8 \pm 1,2$ & $2,5 \pm 1,0$ \\
\hline
\end{tabular}

Legenda: Média \pm DP; CVF: capacidade vital forçada; VEF1: volume expiratório forçado no primeiro minuto. Os valores estão expressos em valores absolutos (L) e percentual (\%).

Fonte: Dados da pesquisa. 


\section{Discussão}

No último século, a ansiedade tem aumentado expressivamente, sendo fequentemente encontrada em idosos. A ansiedade-estado está ligada a um momento ou uma situação particular, que cause um estado emocional transitório, enquanto a ansiedade-traço está relacionada a características individuais e à personalidade de cada um (18-20). No presente estudo, verificou-se que as idosas do grupo G1 apresentaram ansiedade-estado e não apresentaram ansiedade-traço, ao contrário das idosas do grupo G2. Possivelmente esses dados mostram que as idosas do grupo G2 possuem a ansiedade como característica pessoal, ao contrário do grupo G1, que apresentou ansiedade apenas no momento da avaliação.

Com o envelhecimento, ocorre uma diminuição das pressões respiratórias máximas e estima-se que um indivíduo com 60 anos necessite despender 20\% mais força num determinado nível de ventilação do que um indivíduo com vinte anos. Um estudo recente mostrou que as idosas praticantes de atividade física apresentaram um aumento significativo nas pressões respiratórias máximas quando comparadas às sedentárias, concluindo que a atividade física regular pode também reverter ou atenuar a deterioração normal da função respiratória $(21,22)$. Esses dados corroboram com nossos achados, uma vez que as participantes do grupo G1 apresentaram os maiores valores de PEmáx.

A normalidade da cirtometria em um adulto jovem saudável é em torno de $7 \mathrm{~cm}$ de mobilidade torácica. Em decorrência do envelhecimento, ocorrem modificações da estrutura torácica, o que induz a uma limitação na capacidade fisiológica, diminuição da complacência da parede do tórax e da mobilidade dos músculos diafragmáticos e intercostais (23, 24). Em nosso estudo, todas as idosas de ambos os grupos apresentaram grau de mobilidade torácica abaixo dos parâmetros de normalidade.

As participantes do grupo G1 apresentaram aumento da mobilidade basal em relação às do grupo $\mathrm{G} 2$, as quais apresentaram maior mobilidade xifoide. 0 aumento da mobilidade basal encontrada nas idosas praticantes de dança gera benefícios como elevação e distensão do diafragma, com maior atuação dessa musculatura, o que leva a uma melhor respiração (25).

0 envelhecimento gera alterações funcionais, entre elas o aumento da capacidade residual funcional e do volume residual, bem como a diminuição da capacidade vital forçada, do volume expiratório forçado no primeiro segundo e da ventilação voluntária máxima (26). Isso é diferente do que foi encontrado nas idosas dos dois grupos estudados, em que os valores obtidos para fluxos e volumes respiratórios se encontraram acima dos níveis de referência. Talvez isso seja ocasionado pelas boas condições de saúde das idosas que compuseram a amostra.

A amostra reduzida deste estudo não permitiu uma análise estatística mais apurada. Sugerimos que estudos futuros utilizem uma amostra com mais participantes e que o grupo controle seja retirado de uma seleção com base populacional que possa refletir adequadamente a população do estudo.

\section{Considerações finais}

Observaram-se nas idosas que praticam dança de forma regular menor nível de ansiedade-traço, valores adequados de pressão expiratória máxima e maior valor na cirtometria em nível basal, o que leva a uma melhor dinâmica ventilatória. Isso pode indicar um efeito clínico positivo na qualidade respiratória e no bem-estar emocional, o que demonstra a importância da prática da atividade física por meio da dança de salão para esses indivíduos.

\section{Referências}

1. Ramos LR. Fatores determinantes do envelhecimento saudável em idosos residentes em centro urbano: Projeto Epidoso, São Paulo. Cad Saúde Pública. 2003;19(3): 793-8. doi: 10.1590/S0102-311X2003000300011.

2. Almeida OP. Idosos atendidos em serviço de emergência de saúde mental: características demográficas e clínicas. Rev Bras Psiquiatr. 1999;21(1):12-8.

3. Castillo ARGL, Recondo R, Asbahr FR, Manfro GG. Transtornos de ansiedade. Rev Bras Psiquiatr. 2000; 22(Suppl. 2):20-3.

4. King AL, Valença AM, Nardi AE. Hiperventilação. A terapia cognitivo-comportamental e a técnica dos exercícios de indução dos sintomas no transtorno de pânico. Rev Port Pneumo. 2008;14(2):303-8.

5. Ide MR, Caromano FA, Dip MAVB, Guerino MR. Exercícios respiratórios na expansibilidade torácica de idosos: exercícios aquáticos e solo. Fisioter Mov. 2007; 20(2):33-40. 
6. Francisco PMSB, Donalisio MR, Barros MBA, Cesar CLG, Carandina L, Goldbaum M. Fatores associados à doença pulmonar em idosos. Rev Saúde Pública. 2006;40(3):428-35.

7. Franchi KMB, Monteiro LZ, Almeida SB, Pinheiro, MHNP, Medeiros AIA, Montenegro RM, et al. Capacidade funcional e atividade física de idosos com diabetes tipo 2. Rev Bras Ativ Fisic e Saud. 2008;13(3): 158-66.

8. Neri AL, Freire SA. E por falar em boa velhice. Campinas: Papirus; 2003.

9. Nóbrega ACL, Freitas EV, Oliveira MAB, Leitão MB, Lazzoli JK, Nahas RM, et al. Posição oficial da SBME e da Sociedade Brasileira de Geriatria e Gerontologia: Atividade física e saúde no idoso. [acesso 18 nov. 2010]. Disponível em: http://www.saudeemovimento.com. $\mathrm{br} /$ conteudos/conteudo_frame.asp?cod_noticia $=480$.

10. Romano P, Bankoff ADP. A influência da respiração na melhora da técnica de dança clássica. Movimento \& Percepção. 2009;10(14):122-33.

11. Bocalini DS, Santos RN, Miranda MLJ. Efeitos da prática de dança de salão na aptidão funcional de mulheres idosas. Rev Bras Ciênc Mov. 2007;15(3):23-9.

12. Voorrips LE, Ravelli PC, Dongelmans PCA, Deurenberg P, Van Staveren WA. A physical activity questionnaire for the elderly. Med Sci Sports Exerc. 1991;23(8): 974-9.

13. Spielberger CD, Gorsuch RC, Lushene RE. Manual for the state-trait anxiety inventory. Palo Alto: Consulting Psychologists Press; 1970.

14. Sociedade Brasileira de Pneumologia e Tisiologia: II Consenso Brasileiro sobre Doença Pulmonar Obstrutiva Crônica (DPOC). Caracterização da Doença Pulmonar Obstrutiva Crônica (DPOC) - Definição, Epidemiologia, Diagnóstico e Estadiamento. J Bras Pneumol. 2004;30(5):10-4.

15. Pereira CAC, Sato T, Rodrigues SC. Novos valores de referência para espirometria forçada em brasileiros adultos de raça branca. J Bras Pneumol. 2007;33(4): 397-406.

16. American Thoracic Society/European Respiratory Society. ATS/ERS Statement on Respiratory Muscle Testing. Am J Respir Crit Care Med. 2002;166(4):518-624.
17. Paulin E, Brunetto AF, Carvalho CRF. Efeitos de programa de exercícios físicos direcionado ao aumento da mobilidade torácica em pacientes portadores de doença pulmonar obstrutiva crônica. J Pneumol. 2003;29(5):287-94.

18. Ferreira CL, Almodes KM, Braga LP, Mata ANS, Lemos CA, Maia EMC. Universidade, contexto ansiogênico? Avaliação de traço e estado de ansiedade em estudantes do ciclo básico. Ciência \& Saúde Coletiva. 2009; 14(3):973-81.

19. Byrne G. What happens to anxiety disorders in later life? Rev Bras Psiquiatria. 2002;24(1):74-80.

20. Oliveira KL, Santos AAA, Cruvinel M, Néri AL. Relação entre ansiedade, depressão e desesperança entre grupos de idosos. Psic Estudo. 2006;11(2)351-9.

21. Azeredo CAC. Técnicas para o desmame no ventilador mecânico. São Paulo: Manole; 2002.

22. Gonçalvez MP, Tomaz CAB, Cassiminho ALF, Dutra M. Avaliação da força muscular inspiratória e expiratória em idosas praticantes de atividade física e sedentárias. R Bras Cien Mov. 2006;14(1):37-44.

23. Carvalho AR, Butzge DM, Bianchini LC, Rocco PF, Rodrigo ACA, Maso GCD, et al. Influência de técnicas manuais na força muscular respiratória, capacidade funcional e mobilidade do gradil costal de idosos. Fit Perf J. 2008;7(5):338-44. doi:10.3900/fpj.7.5.338.p.

24. Guimarães RM, Cunha UGV. Sinais e sintomas em geriatria. Rio de Janeiro: Atheneu; 2004.

25. Azeredo CAC. Fisioterapia respiratória. São Paulo: Manole; 1984.

26. Assis EL, Rabelo HT. Percepção da capacidade funcional de mulheres idosas praticantes de hidroginástica. MOVIMENTUM - Revista Digital de Educação Física Ipatinga, 2006;1. [acesso 20 nov. 2010]. Disponível em: <http://www.unilestemg.br/movimentum/Artigos_ V1N1_em_pdf/movimentum_assis_elizetelopes.pdf>.

Recebido: 02/12/2010

Received: 12/02/2010

Aprovado: 24/05/2011

Approved: 05/24/2011 